\title{
IL FAUT APPELER UN « SHAD » UN SHAD
}

\author{
Marc SOURDOT \\ Université Paris Descartes
}

\begin{abstract}
Borrowing English words is common to a number of jargons. Fishing jargon, especially that of sport fishing, doesn't escape this rule. But is English the only source of lexical creation? Doesn't it depend on all sorts of discourses such jargons take into account? In other words is there only one jargon for fishing? In order to answer these two questions: the origin of borrowings on the one hand, and on the other hand, either the unity or diversity of one or more jargons involving fishing, I'll rely on my experience as a fisherman/narrator (describer) and on the text of a recording of a fisherman in the middle of a fishing competition.
\end{abstract}

\section{RAPPEL : ARGOTS ET JARGONS}

Il n'est peut-être pas inutile de rappeler ce que nous entendons par « argot » et « jargon ». Nous avons avancé (Sourdot, 2002 : 29) que ces deux domaines se distinguaient essentiellement au travers de critères fonctionnels, et en premier lieu par le caractère cryptique qui explique l'émergence et le développement d'un argot. Si argot et jargon peuvent avoir en commun une dimension identitaire et ludique, l'argot, seul, relève de cette fonction cryptique qui permet au groupe de réserver l'information à ses seuls membres.

Ce qui différencie également un jargon d'un argot, c'est ce que l'on peut appeler la fonction « économique » du premier qui vise à l'efficacité, c'est-à-dire à la précision et à la brièveté dans l'échange.

Ce qu'il faut aussi noter, c'est que ces critères fonctionnels ont des conséquences directes sur les procédés formels utilisés par les uns et les autres. Ainsi l'argot fera un usage plus fréquent de l'aphérèse (tos pour matos) qui masque le sens 
tout en raccourcissant le mot, alors qu'un jargon se contentera, si l'on peut dire, de l'apocope (matos pour matériel) tout aussi " économique » mais sans souci « cryptique ».

N'oublions pas également que, mis à part des témoignages directs, mais rares, recueillis à l'intérieur de groupes argotisants (p. ex. Neury, $1996: 156)$, on se réfère généralement à ce que Denise François appelait « l'argot commun », c'està-dire à ces unités qui ont quitté le cercle étroit des usages cryptés pour se répandre dans la langue commune (François-Geiger, $1991: 7$ ).

Ajoutons enfin, dans cette introduction, que jargon et argot ne sont utilisés, et donc identifiables, qu'à l'intérieur de groupes bien délimités et dans des situations précises. La première conséquence, et non des moindres, c'est qu'un terme de la langue courante peut prendre, dans une discussion entre pêcheurs, un sens bien précis et très différent de cette langue de tous les jours. Ainsi le mot cuillère désigne, pour les pêcheurs, un leurre métallique d'usage courant. À l'observateur de noter ces emplois avant de pouvoir en rendre compte.

\section{Pourquoi l'anglais?}

Il y a bien longtemps que des termes anglais sont utilisés dans le vocabulaire des pêcheurs de loisirs, dits aussi «pêcheurs sportifs ». La première raison en est que les Anglais furent les premiers à considérer la pêche comme un « sport », au sens britannique du terme, à codifier ses usages et à les exporter, par la pratique et la littérature y afférente, dans des pays où la pêche n'était encore qu'une activité « alimentaire ». Ce qui est valable en France l'est également, par exemple, en Allemagne ou Norvège.

Un des premiers ouvrages de pêche, A Treatyse of Fysshynge wyth an Angle (Traité de Pêche à la Ligne) de Dame Juliana Berners date de 1496. Le parfait pêcheur à la ligne d'Isaac Walton date de 1653 et a été traduit et réédité des centaines de fois.

En conséquence, les techniques et le matériel de pêche furent, de longue date, l'apanage des Anglais qui, à la fin du XIX ${ }^{\mathrm{e}}$ siècle furent concurrencés par les Américains qui, désormais, font référence en matière de pêche sportive.

L'antériorité de la pratique n'explique pas tout : la puissance économique et le rayonnement en matière de sport de l'Angleterre au XIX ${ }^{\mathrm{e}}$ siècle puis celle des USA par la suite sont des facteurs tout aussi pertinents. Ceci explique, par exemple, pourquoi les pêcheurs à la mouche français, les «moucheurs », utilisent des mesures en pieds et en pouces pour la longueur de leurs cannes, voire des poids en grains pour peser leurs lignes, termes traduits certes mais relevant d'un autre système de mesure. 
On peut, dès lors, s'interroger sur la non traduction de beaucoup de mots et de tournures. Le fait est que certaines traductions pourraient sembler ridicules. Ainsi le rédacteur en chef de la revue La Pêche et les Poissons avait intitulé l'un des ses éditoriaux «Quiver-tip ou bout-qui-tremble ? » indiquant par là que la traduction du premier terme par le second avait quelque chose de ridicule pour désigner une technique de pêche utilisant un scion, la partie terminale, le bout de la canne, très souple, comme indicateur de touche quand le poisson le faisait trembler.

\section{IL FAUT APPELER UN SHAD UN SHAD}

Mais cette crainte, ou ce risque, du ridicule s'inscrit dans un mouvement plus large, celui qu'il est convenu d'appeler « l'économie linguistique » qui privilégie le « rendement » d'un mot ou d'une tournure. En d'autres termes, est « économique » ce qui permet de communiquer le maximum d'informations avec le minimum de moyens. La brièveté, comme le soulignait déjà Henri Frei dans sa Grammaire des Fautes il y a un siècle (Frei, 1929 : 125), en est un rouage essentiel même s'il n'est pas le seul.

On comprendra, dès lors, qu'un faisceau de raisons peut expliquer l'adoption par les pêcheurs de ces termes anglais, venus d'outre-Manche ou d'outreAtlantique : l'origine du concept ou du produit importé avec son appellation et l'absence de traduction liée au coût comportemental que cela induirait. Ainsi lors de l'apparition de nouveaux leurres en matières synthétiques souples utilisés pour la pêche des carnassiers, truites et brochets par exemple, l'appellation générique, en français, leurre souple s'est-elle imposée. Mais quand les modèles de ces leurres se sont, très vite, multipliés, il a fallu, également, multiplier les appellations.

Et plutôt que d'utiliser leurre souple en forme de poisson pour différencier ce modèle d'un autre, l'appellation shad s'est imposée. Et les pêcheurs d'aujourd'hui n'ont pas l'impression d'employer un anglicisme avec ce vocable qui, de surcroît, correspond dans sa prononciation aux habitudes de notre langue. C'est pourquoi on ne peut faire autrement que d'appeler un shad un shad.

Si l'on insiste sur l'importance des emprunts à l'anglais dans le monde de la pêche, c'est que les autres langues, à de rares exceptions près, ne sont pas sources d'emprunts. Nous pouvons en citer deux de mémoire : Pater-noster et Rigoletto, ce dernier désignant un petit flotteur indicateur de touches d'une marque commerciale italienne. Quant au premier, nous rappellerons, pour mémoire, l'étymologie malicieuse qu'attribue Michel Duborgel au pater-noster, émerillon triple souvent utilisé pour la pêche du brochet : «Ce nom lui a été donné parce que là où il est utilisé, un brochet peut faire son dernier pater noster » (Duborgel, 1974 : 532). 
Tout aussi rares sont les cas d'anglicismes qui ont été francisés, alors qu'ils étaient déjà installés dans l'usage des pêcheurs. Je citerai le terme bouillette qui a remplacé le mot boilie de l'anglais pour désigner ces petites billes d'appâts utilisées dans les pêches modernes de la carpe. La proximité phonique et sémantique ainsi que la brièveté des deux termes peuvent expliquer le succès de cette traduction initiée par Henri Limouzin, rédacteur influent d'une revue halieutique et angliciste par ailleurs. En revanche, le terme graciation, pourtant utilisé par les pêcheurs du Québec, qui désigne le fait de remettre un poisson à l'eau, n'a pu remplacer l'expression no kill utilisée par les pêcheurs français, la brièveté de ce terme expliquant sans doute son succès.

\section{Du français de tous les Jours, MAIS Pas SEUlement}

Il peut parfois être difficile pour le descripteur/pêcheur de se rendre compte qu'il utilise un jargon que les non initiés ne comprennent pas forcément. C'est que toute une partie du vocabulaire de la pêche - et c'est vrai pour tous les jargons - provient de la langue de tous les jours mais prend, pour le domaine concerné et ses utilisateurs, un sens différent.

C'est le cas par exemple de cuillère pour désigner un leurre métallique utilisé pour tromper les poissons. Une tournante sera une cuillère à mouvement rotatif alors qu'une ondulante sera une cuillère à mouvement ondoyant. On notera au passage l'abréviation fréquemment utilisée dans un jargon. Pour le pêcheur à la mouche une queue de rat désigne la partie terminale de la ligne, le bas-de-ligne, de diamètre décroissant.

Mais écoutons plutôt ce court extrait d'enregistrement d'un pêcheur au coup de compétition nous parler de sa pratique :

Au concours de Villeneuve, je suis tombé en plein mitard, alors là aucune chance malgré mes panachés : bricheton, terreau, bloche. À Villetaneuse j'ai touché l'as et l'aile amont dans la deuxième manche. Le mitard ça vaut pas un clou, je me suis tapé une rondelle. À Villetaneuse à 5 minutes de la fin je décroche un plateau et je loupe la gagne. Quand la pêche est archiduraille, faut sortir la bijouterie : mitraillette et le 28 sur 6 . Les petites ablettes, $y$ en faut beaucoup pour faire la douzaine !

Il n'y a effectivement pas d'emprunts à l'anglais dans ce court extrait. Il faut préciser qu'il s'agissait, à l'époque, d'un pêcheur au coup « à la grande canne », 
pratique traditionnelle des pêcheurs français. Il en aurait été autrement si je m'étais adressé à un pêcheur de compétition « au moulinet » au vocabulaire plus influencé par l'anglais.

Il y a certes, ici, du français de tous les jours, mais il semble difficile, pour un non-pêcheur, de comprendre ce jargon qui emprunte beaucoup à l'argot commun et dont chaque terme, ou presque, demande explication.

Le vocabulaire du "français de tous les jours », d'abord, prend ici des significations bien particulières. Le « concours » est une compétition de pêche au coup ; « tombé » est employé à la place de "placé »; un " panaché » est un mélange d'esches ; «terreau » est l'abréviation de « ver de terre » et l'« as » est le poste $\mathrm{n}^{\circ} 1$, le meilleur en compétition. Un " plateau » est un poisson, une grosse brème ; la " bijouterie » relève du matériel de précision. Une « mitraillette » est une canne très courte, utilisée pour les pêches de vitesse.

Quant à l'argot commun, il demande aussi, dans ce contexte, éclaircissement. Le « mitard » est la position centrale lors de la compétition, au milieu des autres pêcheurs, la plus mauvaise. Le « bricheton » est une variété de pain utilisé comme esche ; un «bloche » est le terme utilisé pour asticot. « Se taper une rondelle » est l'équivalent de « être capot», c'est à dire ne rien prendre du tout, terminer avec 0 poisson. La " gagne » c'est la victoire; « archiduraille » peut être paraphrasé par « très difficile ». Quant à « 28 sur 6 », c'est l'abréviation de « un hameçon $n^{\circ} 28$ sur une ligne d'un diamètre de 6 centièmes ».

Je pense avoir suffisamment « traduit » ce texte pour les lecteurs non-pêcheurs, mais je n'en suis pas sûr ! Car certaines tournures me sont tellement familières que je n'en vois pas toutes les difficultés. C'est le problème du descripteur/pratiquant que j'évoquais plus haut.

Que ce soit dans l'utilisation du français de tous les jours ou dans des usages teintés d'argot commun, il y a souvent une volonté ludique sous-jacente qui vient renforcer la connivence entre participants d'une même activité. C'est ainsi que l'on peut interpréter la dernière phrase, apparemment absurde, de notre petit corpus, ou encore l'emploi de « mitard » qui rappelle la cellule d'isolement en prison.

\section{MieuX ABORder LeS JARgons}

Ces précisions concernant le jargon des pêcheurs nous amènent, à partir de notre expérience, à faire quelques remarques pour essayer d'améliorer la prise en compte et la description des jargons. Le premier point nous semble concerner la 
position du descripteur vis-à-vis de l'objet à décrire. Il doit, certes, être au fait de la pratique prise en compte et connaître le milieu dans lequel elle s'exerce ainsi que les acteurs et leur environnement. Mais le descripteur doit veiller à prendre un certain recul, à garder une certaine distance, s'il ne veut pas risquer de passer à côté de certains traits de jargons. Nous avons évoqué le cas de ces mots de la langue courante qui, dans le jargon, prennent un sens bien différent, comme cuillère par exemple. J'ai interrogé plusieurs pêcheurs en leur demandant s'ils considéraient, parmi d'autres mots présentés, si cuillère leur semblait relever du jargon de la pêche : tous ont répondu par la négative, contrairement à ondulante et tournante. Immersion, certes, mais immersion distanciée donc !

Doit aussi être prise en compte la dimension temporelle. Tout va très vite quand on a affaire à un jargon à la fois technique et de loisirs, comme la pêche. La dimension économique, commerciale, de l'activité fait que les changements de pratiques et, surtout, de matériel sont permanents. La pêche aux leurres d'aujourd'hui n'a plus rien à voir avec celle des années 50 , et le vocabulaire employé a tout naturellement évolué.

Toute aussi importante nous semble être la question « unité/diversité ? 》du jargon de la pêche. En d'autres termes, sommes-nous en présence d'un jargon unique ou de divers jargons correspondant à autant de techniques?

Certes, la pêche au coup traditionnelle, à la grande canne, est très différente de la pêche au coup au moulinet, mais elles ont suffisamment de traits communs pour qu'on puisse trouver, dans la mise en mots de leurs pratiques, suffisamment de vocabulaire similaire et en faire deux variantes du même jargon. Mais qu'en est-il si l'on rapproche les pêches au coup de la pêche à la mouche et de la pêche aux leurres?

Si l'on peut, à force de descriptions de plus en plus minutieuses, mettre en évidence des traits distinctifs à telle ou telle technique de pêche, on relèvera également, et inversement, un certain nombre de traits partagés qui nous permettront de parler d'un « jargon commun » de la pêche de loisirs.

\section{Références bibliographiques}

BERNERS, Juliana (2015 [1496]), A Treatyse of Fysshynge wyth an Angle, London, Classic reprints series.

FRANÇOIS-GEIGER, Denise (1991), « Panorama des argots contemporains », Langue française, $n^{\circ}$ 90, pp. 5-9.

DUBORGEL, Michel (1974), Traité pratique de la pêche en eau douce, Paris, Éditions Morgan. 
FREI, Henri (1971 [1928]), La Grammaire des fautes, Genève, Republications Slatkine (Paris, P. Geuthner pour la $1^{\text {ère }}$ éd.).

NEURY, Philippe (1996), « À propos d'argot : essai d'enquête et d'analyse », BULAG, ${ }^{\circ}$ Hors Série, Colloque de Cérisy, 10-17 août 1994, pp. 158-164.

SOURDOT, Marc (2002), «L'argotologie : entre forme et fonction », La Linguistique, vol. 38, pp. 25-39.

WALTON, Isaac (2017 [1653]), Le Parfait Pêcheur à la ligne, Grenoble, Jérôme Millon. 